\title{
Del campo a la Universidad: aspiraciones de jóvenes de familias agricultoras de Chile
}

\author{
From the Countryside to the University: Aspirations of Young People from \\ Agricultural Families in Chile
}

\author{
Hernán Allendes Sandoval y Enrique Rivera García ${ }^{1}$
}

\section{Resumen}

En Chile se observan importantes transformaciones a nivel familiar en el medio rural. Los y las jóvenes de hoy responden al trabajo agrícola de forma distinta a sus progenitores, debido principalmente a las aspiraciones que surgen en relación a la apertura de la educación superior en el país. En este contexto de cambio social, la investigación de la que procede este artículo se plantea abordar dos cuestiones: la dimensión subjetiva que subyace al abandono de la agricultura en hijos e hijas de familias agrícolas, y la configuración de las aspiraciones intergeneracionales a partir de la apertura de la educación superior. La investigación se realizó a través de entrevistas semi-estructuradas a familias agricultoras en la región de Valparaíso. Los resultados muestran que fenómenos como el envejecimiento de la población agrícola y las aspiraciones de la población joven hacia la educación superior han promovido un proceso paulatino de abandono de la agricultura.

\section{Palabras claves}

Aspiraciones, jóvenes, familia, agricultura, educación superior.

\section{Abstract}

In Chile, initiated in the dictatorship of Pinochet in 1973, there are important transformations at the family level in rural areas. Young people today respond to agricultural work very differently from their parents, mainly due to the aspirations that arise in relation to the opening of higher education in the country. In this context of social change, the research from which this article proceeds addresses two issues: 1) the subjective dimension that underlies the abandonment of agriculture in the sons and daughters of agricultural families, and 2) the configuration of intergenerational aspirations From the opening of higher education. The research was conducted through semi-structured interviews with farming families in the Valparaíso region. The results show that phenomena such as the aging of the agricultural population and the aspirations of the young population towards higher education have promoted a gradual process of abandonment of agriculture.

\section{Keywords}

Aspirations, family, agriculture, higher education. 


\section{Cambios sociales en contextos rurales: hacia un estado de la cuestión}

A partir de la década del ochenta en toda Latinoamérica se evidencia un proceso de transformaciones a nivel productivo. Con el advenimiento de los regímenes dictatoriales, una serie de políticas de Estado, de inspiración neoliberal, fueron aplicadas a la agricultura. Estas políticas buscaban el desmantelamiento de las reformas agrarias que los distintos países de la región habían implementado con anterioridad, comenzando un proceso que favoreció a la agro-industria, pero empobreció y perjudicó a la pequeña agricultura familiar. Este fortalecimiento del capitalismo en la agricultura, en su forma neoliberal, en el caso del país en que se centra este artículo, Chile, fue llamado proceso de contra-reforma (Kay, 1997), debido a los cambios que produjo con respecto a la etapa precedente, donde la modernización de la agricultura a través de la desaparición de la hacienda y latifundio, y la tenencia de tierra de los campesinos era fundamental para beneficiar la industrialización interna, bajo el plan de promoción de industria nacional de la época (Valdés y Foster, 2015).

Por su parte, en Chile, durante los últimos veinte años, se ha insistido en la profundización de las políticas impuestas durante la dictadura de Pinochet, que incluían apoyo a la agro-exportación y al empresariado agrícola (Gomez y Echeñique, 1988; Kay, 1997), y encuentran su inspiración en la teoría políticoeconómica del neoliberalismo. Según Harvey (2007), esta teoría busca el bienestar humano a través del «libre desarrollo de las capacidades y de las libertades empresariales del individuo» (p.8).

Estas políticas produjeron una asalarización de antiguos campesinos beneficiados con la reforma agraria, y un empeoramiento en las condiciones laborales de los trabajadores agrícolas, lo que se conoce como una forma de «descampesinización hacia abajo» (Kay, 1997; Gonzalez, 2003).

Estos fenómenos ya mencionados en Latinoamérica han estado estrechamente vinculados a la denominada cuestión campesina, de la que se desprenden dos vertientes entorno al análisis de la coexistencia del campesinado y el capitalismo. Según Vivanco y Flores (2005), la visión campesinista se enfoca a la subsistencia y resistencia del campesinado y la producción agrícola familiar dentro del capitalismo. Por su parte, la visión descampesinista postula una desaparición progresiva e inevitable de estas formas de organización económica y cultural bajo el sistema de producción capitalista. En la práctica, estas corrientes no son excluyentes entre sí y, más que obedecer a una voluntad particular, son el estudio de distintos procesos simultáneos (Hernández, 1994)

En sentido opuesto, en estudios empíricos hechos en Argentina, también se reconoce una descampesinización «hacia arriba», en donde hay un paso de campesinos a productores logrando, con ello, un cierto grado de semi-capitalización en la producción agrícola familiar (Archetti, 1977; Murmis, 1991). En suma, se trata de una diversificación de las formas de desarraigo de la tradicional producción campesina, experimentado tanto en Chile como en toda Latinoamérica en general.

Durante este período de transformaciones en la agricultura, algunos estudios han prestado atención a la juventud rural, describiendo procesos de creciente desarraigo de la institución familiar tradicional, principal sostén de la producción agrícola de pequeños y medianos productores campesinos, además del creciente ingreso de las mujeres al mercado laboral y la presencia de mayor migración juvenil en sectores urbanos. Estos elementos muestran importantes cambios en la composición y dinámica interna de las familias rurales (Díaz y Duran, 1988; Bertrán y Corbalán, 1990; De La Maza, 1993; CEPAL, 1994; Colodro, 1995). 
Otro elemento que ha estado presente recientemente en los estudios sobre la juventud rural, tiene que ver con sus aspiraciones en tanto estudiantes, como respuesta a una creciente urbanización hacia el campo, proximidad que ha provocado un impacto en el estilo de vida campesino, principalmente en la adquisición de valores y perspectiva asociados a lo urbano (estudios, consumo, bienes y servicios). Éstas han tenido mayor presencia desde los años ochenta y noventa en Estados Unidos y en Europa principalmente (Sherwood, 1989; Quaglia y Cobb, 1996; Gutman y Akerman, 2008). En este contexto, las aspiraciones de jóvenes en zonas rurales y, con ello, el abandono de la agricultura, quedan relacionados por la posibilidad de estudiar en la universidad. En este sentido, las aspiraciones de los y las jóvenes son altas ante la expectativa de tener una mejor calidad de vida. Ahora bien, en el contexto neoliberal actual, la inseguridad que despierta la posibilidad de éxito o fracaso de estas aspiraciones es psicologizada por la población juvenil con una alta carga emocional, esto es, cada joven va a asimilar su éxito o fracaso como una responsabilidad individual, y no tanto considerando también los condicionantes estructurales de ese éxito/fracaso (Cairns, 2013).

En Chile, ante la masificación de la educación superior a través de la creación de instituciones privadas de educación (Brunner, 2015), las aspiraciones y expectativas de jóvenes rurales son altas, principalmente porque suponen una mejora de las condiciones laborales a pesar de la profunda segmentación de la educación chilena (Sepúlveda y Valdebenito, 2014). Es en este punto donde se incorpora el estudio de las relaciones intergeneracionales y la tendencia progresiva a la «desagrarización» y «desruralización» en las generaciones más jóvenes. Estas tendencias se refieren a la disminución de la pequeña y mediana producción agrícola en zonas rurales, y a la disolución de la antigua frontera rural-urbana, respectivamente. Sin embargo, a pesar de que existe una desruralización en términos de proyectos de vida y empleos en la población joven, el aumento de servicios, recreación y transporte en zonas rurales, permite que los jóvenes no sean «expulsados» a la ciudad, sino que mantengan su residencia en el campo (Valdés y Rebolledo, 2015). Es decir, que el abandono progresivo del trabajo agrario por parte de la generación más joven no conlleva en sí un abandono similar del entorno rural, puesto que lo que se abandona son los aspectos simbólicos de la proyección en el campo, que se mantienen en cierta medida por la permanencia de la residencia en este territorio.

Así, pues, en el marco de este análisis, la investigación de la que procede este artículo pretende actualizar el debate contemporáneo sobre lo rural y su continuidad en el trabajo agrícola-familiar en Chile. En concreto, este artículo busca comprender los cambios actuales en torno a los proyectos de vida de pequeños productores agrícolas en la región de Valparaíso. La tesis de partida es que fenómenos sociales simultáneos, como son el envejecimiento de la población agrícola chilena y las aspiraciones de la población joven con respecto a la educación superior, han provocado un proceso paulatino de abandono de la agricultura en el país. En consecuencia, a través de un enfoque cualitativo de carácter exploratorio, este artículo busca analizar dos aspectos relevantes, a saber: 1) los elementos subjetivos que subyacen al proceso de abandono de la agricultura en hijos e hijas de familias agricultoras, y 2) la formación de aspiraciones intergeneracionales en torno a la educación superior. La investigación se ha realizado mediante la técnica de producción de datos cualitativos que es la entrevista semi-estructurada, que se ha realizado a padres/ madres e hijos/as de familias dedicadas a la agricultura. La muestra contempla siete familias residentes en las comunas de Quillota, Limache y Olmué, en la región chilena de Valparaíso tradicionalmente agrícola. El objetivo ha sido explorar los procesos subjetivos de la población joven de esta región, entendiéndolos como elementos que configuran sus aspiraciones, y cómo éstos cristalizan en estrategias y proyecciones individuales y familiares. 


\section{Aproximación a las nociones de subjetividad y aspiraciones}

\subsection{Aproximación al concepto de subjetividad}

La subjetividad es un concepto ampliamente tratado por las Ciencias Sociales. Una de sus acepciones, que será empleada en esta investigación, señala que la subjetividad surge como expresión de múltiples factores, constituyéndose a partir de códigos simbólicos que nos construyen y nos permiten, además, construir la realidad social objetivada (Vargas, 2003). Esa realidad construida reproduce y manifiesta símbolos que adquieren sentido a través de la comunicación que, en nuestros tiempos, implica una sobrecargada forma de proponer, imponer y resignificar valores y creencias, como también percepciones y aspiraciones. En este intercambio, «la subjetividad es un campo de significaciones, un conjunto de concepciones del mundo y de la vida, donde se entrelazan diversos niveles de comprensión y aprehensión de una cosmovisión» (Gil, 2008: 642).

Esta tarea no solo implica identificar qué rasgos simbólicos adquiere el mundo, sino también identificar la posición del sujeto en el entramado social, ya que comunicar las percepciones, creencias o aspiraciones, implica una posición concreta en las relaciones que se establecen para y con los otros en contextos ambientales diversos (Morin, 1998). En este contexto, entendemos que la subjetividad se construye mediante dos procesos complementarios: la socialización, que hace posible la reproducción del orden social, y la subjetivación, que comprende una construcción más activa por parte del sujeto agente (Venegas, 2017). Cabe destacar que esto último asume una relevancia especial para nuestro objeto de estudio, pues indica que los primeros círculos afectivos y de socialización están relacionados al campo subjetivo, sin embargo, es una relación donde se deja entreabierta la posibilidad de una transformación generacional, pensando en la familia como espacio socializador temprano.

La subjetividad surge como campo de posibilidad que tiene un sujeto inmerso en estructuras macro y meso-sociales, externas y coercitivas pero que, en el nivel micro-social de la acción y la interacción, se configura como medio con la posibilidad de transformarse en el proceso a través del cual el sujeto construye su identidad (Connell, 1987; Venegas, 2017). Así pues, «La socialización es un proceso de naturaleza macro-sociológica, pero tiene lugar en el nivel micro-sociológico, poniendo en relación estructura y acción [...] En su formulación más original, el individuo internaliza el orden estructural -lo hace suyo-, para reproducirlo mediante la acción» (Venegas, 2017: 25), o desde un punto de vista crítico, transformarlo desde sí, para sí o para otros. En el caso de la investigación de la que procede este artículo, será a partir de la práctica social de la población joven, procedente de familias agricultoras de la zona central de Chile, donde se sitúe el debate entre la práctica reproductora del sistema social neoliberal presente, o la práctica de resistencia al mismo, en virtud del proyecto de vida al que aspira cada joven. Como menciona Venegas, «las prácticas ponen en relación los dos niveles estructurales, el material, que las contextualiza, y el simbólico, que las orienta dentro de ese contexto material [...] las prácticas resultantes de esa sumisión al discurso dominante son, en última instancia, prácticas de reproducción del orden social establecido, al replegarse al mismo» (Venegas, 2017: 26). Ahora bien, también cabe la posibilidad del cambio social, que en nuestro caso se observa en las aspiraciones y proyecciones de corto y mediano plazo en los jóvenes, quienes irrumpen en las familias agricultoras con otros discursos que se vislumbran discordantes con la dimensión simbólica del campo y el trabajo agrícola. En otras palabras, sus prácticas y discursos de resistencia se dan al interior de la familia, pues buscan seguir caminos distintos a los de sus padres, pero si se observan sus aspiraciones podríamos entrever que estas son más bien de reproducción del discurso dominante a nivel macro-social. 
En esta investigación, el nivel macro de análisis se corresponde con la sociedad chilena, en que se observa un proceso de subjetivación entre la población joven definido por políticas fuertemente neoliberales, junto a la opción por la educación superior, que implica emigrar del campo a la ciudad y, con ello, alejarse del quehacer familiar agrícola. El nivel meso-social se corresponde con instituciones de socialización y subjetivación de relevancia para la generación joven que analiza esta investigación, tales como la familia, la producción agrícola (entendida como sistema productivo o económico), el entorno social inmediato (esto es, el medio rural, física y socialmente) y, sobre todo, la universidad (como sistema educativo).

\subsection{Subjetividad y aspiraciones en el contexto neoliberal}

En cuanto al concepto de aspiraciones, se sigue aquí el enfoque de Gutman y Akerman (2008), quienes proponen que las aspiraciones son proyectos y objetivos de vida de las personas, constituyendo un fenómeno de múltiples dimensiones, en el que el factor educacional, si bien es importante, no es necesariamente el principal. Se sugiere también la naturaleza contextual de las aspiraciones, ya que, si bien éstas son formadas a través de la subjetividad de los individuos, también se «dibujan» desde la vida de otras personas situadas alrededor de ellos. De esta forma, la noción de una «aspiración alta» solo se puede afirmar en comparación a otra que es «baja» en un contexto determinado. Por tanto, las aspiraciones serán entendidas aquí como aquellas proyecciones que hacen los y las jóvenes a partir de sus propios procesos de subjetivación, esto eso, de su devenir sujetos agentes o sujetos de su propia acción social (Venegas, 2017), que emergen en contextos de neoliberalización de la vida social en Chile.

Del mismo modo, se puede remarcar la importancia del contexto histórico en el desarrollo de las aspiraciones de esta generación joven en Chile. Un elemento son los cambios históricos experimentados por el mercado laboral y la educación. Otro elemento de cambio, señalado por Gutman y Akerman (2008), es la naturaleza evolutiva de las aspiraciones, pues éstas varían entre la infancia, la adolescencia y la adultez. A esto se suman las influencias externas en la formación de las aspiraciones, siendo el entorno familiar (como agente de socialización primaria) la influencia más importante, sobre todo durante la infancia y adolescencia. Por tanto, las características de las familias tales como el nivel educativo, los ingresos y la clase social son elementos a considerar.

Así pues, cabe poner el foco de atención en el análisis de la subjetividad y las aspiraciones de la población joven en un contexto de políticas neoliberales y sus efectos en la sociedad. Sobre esto, Kate Cairns (2013) desarrolla una investigación con jóvenes estudiantes de zonas rurales en Estados Unidos en que incorpora elementos de la teoría posestructuralista. Cairns analiza la relación entre sus aspiraciones y la producción de subjetividades en la sociedad neoliberal norteamericana. Destaca la 'aproximación individual' de los jóvenes a sus proyectos de vida, recayendo en ellos sus decisiones sobre sus estudios, así como el éxito o fracaso de las mismas. Para la autora, es resultado del discurso neoliberal dominante, que pone el énfasis en el éxito económico de las personas como principal factor de bienestar personal. De esta manera, las decisiones que toman los jóvenes sobre su educación están estrechamente vinculadas a la posibilidad de tener una buena vida y felicidad. Por su parte, Schwiter (2012) defiende que existe una subjetividad neoliberal como tal, expresada en los discursos de los sujetos al imaginar y organizar sus 'proyectos de vida'. Esta individualización de los proyectos vitales está presente en la unicidad de las elecciones tanto personales como laborales, incluso en el seno familiar de jóvenes adultos, en línea con la individualización de los discursos en una sociedad neoliberal. 
Beck (1998) sostiene que la nueva modernidad del neoliberalismo configura una «sociedad del riesgo», donde los sujeto son individualizados sin certezas ni seguridades hacia el futuro, dado la retirada del Estado como garante de protección social.

Estas interpretaciones sobre la subjetividad de corte post-estructuralista siguen el referente de Foucault, quien analiza cómo los discursos y prácticas discursivas son legitimados o deslegitimados por las instituciones políticas que ejercen el poder para establecer normas sobre lo correcto y lo incorrecto. Para Foucault, el discurso indica la relación entre saber-poder, y es a través del discurso donde «tiene lugar la producción social de significado, por medio del cual se produce la subjetividad y se mantienen las relaciones de poder» (Kenway, 1990: p.175). Podemos sostener que la individualización y la concepción del mercado como regulador de la vida humana son discursos y prácticas enseñados y aprendidos, como ocurre también en el campo de la educación y las políticas educativas (Díez-Gutierrez, 2015). Un referente en este sentido lo ofrecen Stephen J. Ball y Deborah Youdell (2008), quienes analizan la privatización de la educación pública como un proceso global, así como en sus formas particulares. Así, a nivel general, hablan de privatización endógena, referida a la transformación de instituciones públicas a un modo privado-empresarial de operar y gestionar la educación, y privatización exógena, que implica la participación de instituciones privadas en establecimientos de educación pública para obtener beneficios. Siguiendo a Foucault, Ball $(2013,2015)$ ha analizado las formas micro-sociales donde se desarrollan las políticas educativas neoliberales. A partir de entrevistas a profesorado de Reino Unido, sostiene que en el contexto del ejercicio del poder político educacional, la subjetividad es un campo clave en la disputa política acerca de la 'verdad', lo 'falso' y lo 'ético' y 'no ético', problematizando la constitución del sujeto consigo mismo y con los otros. En efecto, el sistema neoliberal de educación produce un nuevo sujeto-profesor de acuerdo a las normas que este sistema ejerce. No obstante, el poder es asumido y resistido simultáneamente por los sujetos a través de la "performatividad», o la subjetivación (Venegas, 2017).

Esta doble concepción del poder es clave en el análisis que se propone en este artículo, cuyo objeto de estudio queda delimitado, pues, en el marco de los procesos subjetivos subyacentes a las aspiraciones de las hijas e hijos de familias de agricultores de la región de Valparaíso, en el contexto de la profundización del modelo neoliberal en Chile, y la relación que se establece entre este modelo y los aspectos subjetivos que produce, a saber: la individualización de los proyectos de vida y de las aspiraciones de los sujetos.

\section{Metodología}

Este estudio es de tipo exploratorio-descriptivo. Exploratorio porque se acerca al tema del abandono de la agricultura en cuanto a fenómeno intergeneracional subjetivo; y descriptivo pues específica características de las personas, grupos, sometidos a dicho proceso (Dankhe et al., 1991). De esta manera, este enfoque se justifica en la medida en que permite generar un acercamiento a tal grado que posibilita la generación de información cualitativa válida, en tanto que es otorgada desde, y por, los integrantes de las familias que participan en este estudio. La técnica utilizada para la producción de datos ha sido la entrevista semi-estructurada en profundidad. Según Bourdieu (2010), la entrevista se concibe como una relación social donde se establece un vínculo de confianza entre dos personas, siendo de carácter íntimo y experiencial. Para Guber (1994), la entrevista responde a «una serie de intercambios discursivos entre alguien que interroga y alguien que responde, según pautas de interacción establecidas y aceptadas por las 
partes $(\ldots)$-siendo- una relación comunicativa y productiva de información específica» (p.30). La importancia de este método radica en que permite comprender el espacio social que ocupa otro ser humano mediante una escucha activa y metódica. Se ha optado por el tipo semi-estructurado pues permite abrir nuevos temas importantes para los sujetos de la investigación en la medida en que avanza la entrevista. En cuanto al tipo en profundidad, «pone en relación de comunicación directa cara a cara a un investigador/ entrevistador y a un individuo entrevistado con el cual establece una relación peculiar de conocimiento que es dialógica, espontánea, concentrada y de intensidad variable» (Canales, 2006: 219-220). Para analizar las entrevistas, se ha utilizado el software de análisis cualitativo Nvivo 11, que facilita la categorización y el estudio de tópicos, ideas, temáticas y representaciones en las entrevistas en función de los objetivos de la investigación. Las entrevistas fueron realizadas entre los meses de julio y septiembre del año 2011.

\subsection{Participantes del estudio}

Dado que esta investigación no busca la cuantificación, sino la comprensión del fenómeno estudiado en su complejidad, se ha utilizado lo que se conoce como muestreo selectivo o intencional, donde «cada unidad -o conjunto de unidades- es cuidadosa e intencionalmente seleccionada por sus posibilidades de ofrecer información profunda y detallada sobre el asunto de interés para la investigación» (Martínez y Salgado, 2012: 614).

Los participantes de esta investigación está compuesta por siete familias agricultoras de la región de Valparaíso, en la zona de Quillota, Limache y Olmué, que pertenecen al programa SAT (Servicio de Asesoría Técnica) del INDAP (Instituto de Desarrollo Agropecuario). Este programa busca aumentar las capacidades para mejorar los negocios de los pequeños productores agrícolas, campesinos y sus familias, a través de incentivos y asesorías. Cabe señalar que el programa está destinado al «pequeño productor agrícola», que explota una «superficie no superior a las 12 Hectáreas de Riego Básico, cuyos activos no superen el equivalente a 3.500 Unidades de Fomento, que su ingreso provenga principalmente de la explotación agrícola, y que trabaje directamente la tierra, cualquiera que sea su régimen de tenencia» (Ibíd.: 53).

La categorización de los sujetos sigue los siguientes criterios: 1) generacional, padres/madres e hijos (as, 2) ingreso familiar, 3) nivel educativo, 4) propiedad o no del terreno que se cultiva y 5) enfoque de género. De cada familia se ha entrevistado a dos miembros de generaciones diferentes (progenitor y descendiente). Al observar el ingreso percibido por los grupos familiares participantes, es posible una mejor aproximación al nivel de producción familiar. La tabla 1 muestra el perfil socioeconómico de los sujetos entrevistados. Se ha dividido en tres grupos, siguiendo la encuesta de Caracterización Socioeconómica (CASEN) de 2015, elaborada por el Ministerio de Desarrollo Social de Chile. Los ingresos del tramo superior son mayores a 615.111 pesos chilenos (US803). Por lo tanto, los ingresos aquí considerados en la categoría $\mathrm{A}$, son los únicos que están por debajo de esta línea, mientras que B y C están sobre ella. Sin embargo, si consideramos los promedios de la tabla siguiente, podemos establecer que nuestros participantes categorizados en la letra A se ubican entre el primer y quinto decil (ingresos entre los 113 y 729 US), mientras que B y C pertenece a los tramos inmediatamente superiores (a partir de los 858US). 
Tabla I. Distribución socioeconómica de nuestros entrevistados

\begin{tabular}{cccc}
\hline DECIL & PESOS CL & US $^{2}$ & CATEGORÍAS AQUÍ USADAS \\
\hline I-V & $73.046-467.680$ & $113-729$ & A \\
\hline$V-X$ & $550.954-2.477 .345$ & $858-3.862$ & $B$ \\
\hline$+X$ & +2.477 .345 & 1.098 & $C$ \\
\hline
\end{tabular}

Fuente: elaboración propia con datos de la encuesta CASEN 2015

\section{Tabla II. Perfil socioeconómico de los sujetos entrevistados}

\begin{tabular}{|c|c|c|c|c|c|c|}
\hline \multirow[b]{2}{*}{ SUJETO } & \multirow[b]{2}{*}{ SEXO } & \multirow[b]{2}{*}{ EDAD } & \multicolumn{4}{|c|}{ NIVEL EDUCATIVO } \\
\hline & & & INGRESO FAMILIAR & ED. BÁSICA & ED. SECUNDARIA & PROPIETARIO \\
\hline \multicolumn{3}{|c|}{ Familia Céspedes } & \multirow{3}{*}{ B } & & & \multirow{3}{*}{$\mathrm{Si}$} \\
\hline Padre & M & 57 & & $\mathrm{Si}$ & No & \\
\hline Hija & $\mathrm{F}$ & 22 & & $\mathrm{Si}$ & $\mathrm{Si}$ & \\
\hline \multicolumn{3}{|c|}{ Familia Gaete } & \multirow{3}{*}{ A } & & & \multirow{3}{*}{ No } \\
\hline Padre & M & 49 & & $\mathrm{Si}$ & No & \\
\hline Hija & $\mathrm{F}$ & 23 & & $\mathrm{Si}$ & $\mathrm{Si}$ & \\
\hline \multicolumn{3}{|c|}{ Familia Gomez } & \multirow{3}{*}{ A } & & & \multirow{3}{*}{ № } \\
\hline Padre & M & 64 & & $\mathrm{Si}$ & No & \\
\hline Hija & $\mathrm{F}$ & 21 & & $\mathrm{Si}$ & $\mathrm{Si}$ & \\
\hline \multicolumn{3}{|c|}{ Familia Tapia } & \multirow{3}{*}{ B } & & & \multirow{3}{*}{$\mathrm{Si}$} \\
\hline Padre & M & 57 & & $\mathrm{Si}$ & $\mathrm{Si}$ & \\
\hline Hija & $\mathrm{F}$ & 21 & & $\mathrm{Si}$ & $\mathrm{Si}$ & \\
\hline \multicolumn{3}{|c|}{ Familia Sánchez } & \multirow{3}{*}{ C } & & & \multirow{3}{*}{$\mathrm{Si}$} \\
\hline Padre & M & 47 & & $\mathrm{Si}$ & $\mathrm{Si}$ & \\
\hline Hija & $\mathrm{F}$ & 23 & & $\mathrm{Si}$ & $\mathrm{Si}$ & \\
\hline \multicolumn{3}{|c|}{ Familia Olmedo } & \multirow{3}{*}{ C } & & & \multirow{3}{*}{$\mathrm{Si}$} \\
\hline Padre & M & 45 & & $\mathrm{Si}$ & $\mathrm{Si}$ & \\
\hline Hijo & M & 22 & & $\mathrm{Si}$ & $\mathrm{Si}$ & \\
\hline \multicolumn{3}{|c|}{ Familia Pradenas } & \multirow{3}{*}{ B } & & & \multirow{3}{*}{ No } \\
\hline Padre & M & 66 & & $\mathrm{Si}$ & No & \\
\hline Hijo & M & 22 & & $\mathrm{Si}$ & $\mathrm{Si}$ & \\
\hline
\end{tabular}

Fuente: elaboración propia con datos de la encuesta CASEN 2015.

\section{Análisis y discusión de los resultados}

Esta sección está organizada en dos partes. En la primera, se presentan los temas e ideas más recurrentes en las entrevistas, tal como han sido observados en la codificación de las mismas. En la segunda, se analizan los datos procedentes de las entrevistas, y se discuten los principales resultados en torno al objetivo de esta investigación, a saber: 1) la dimensión subjetiva que subyace al abandono de la agricultura en hijos e hijas de familias agrícolas, y 2) la configuración de las aspiraciones intergeneracionales a partir de la apertura de la educación superior en Chile. Finalmente, se discuten también las implicaciones y posibles líneas futuras de investigación surgidas de este estudio. 


\subsection{Temas más destacados en las entrevistas}

Como puede verse en el Gráfico 1, son las referencias sobre las aspiraciones las que tienen una presencia mayor en las entrevistas, destacando la diferencia en comparación a otros temas emergidos durante la conversación. Recuérdese que la técnica utilizada es semi-estructurada, por lo tanto, existe una mayor libertad de los sujetos de la investigación para que puedan abordar distintas temáticas durante las entrevistas. El gráfico 1 muestra los siete aspectos más recurrentes en el total de entrevistas.

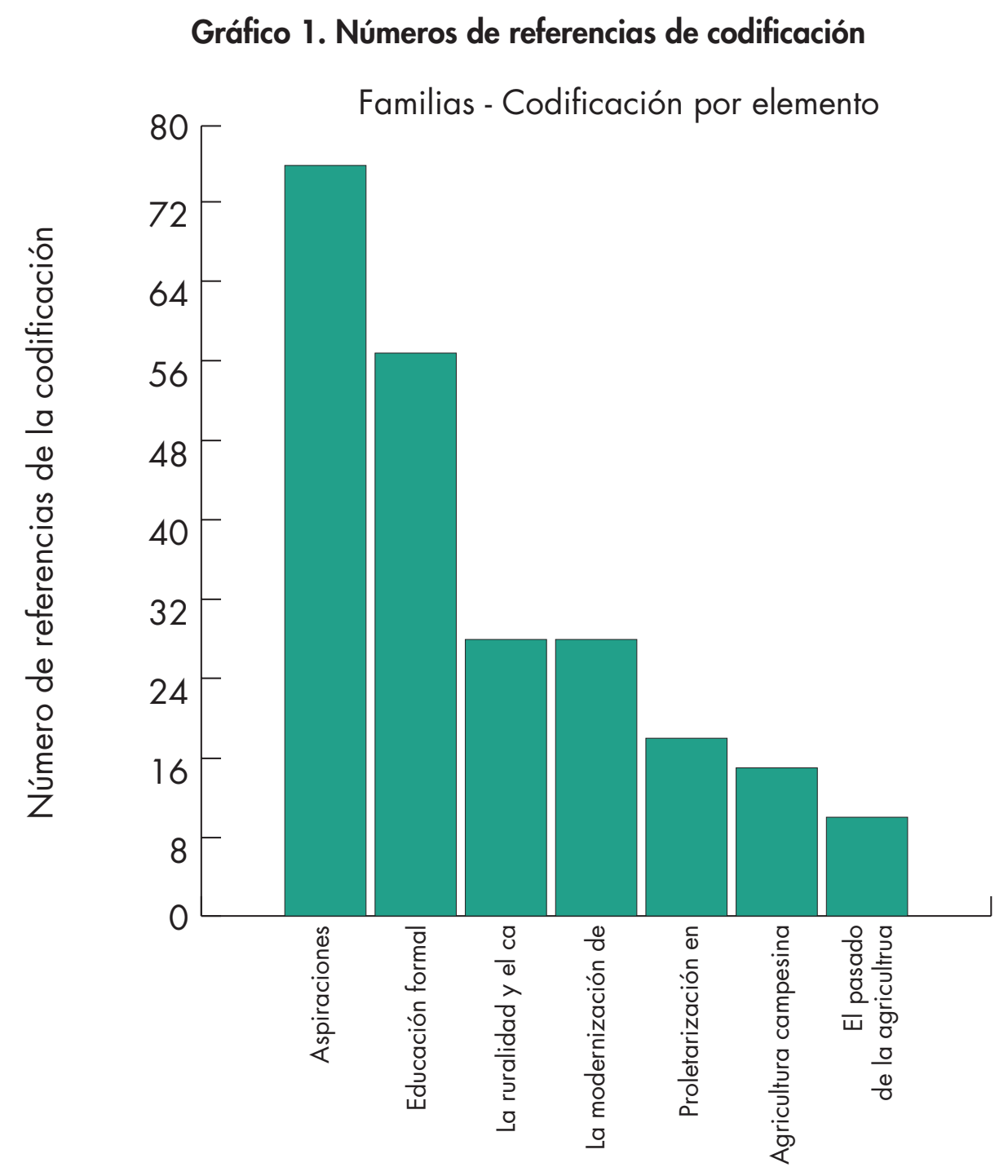

Fuente: elaboración propia con herramienta de codificación Nvivo 11.

\subsection{Cambios generacionales en torno al trabajo agrícola}

En las entrevistas se consta que, a tenor de los sujetos entrevistados, la relación entre trabajo y salario no era beneficiosa para los trabajadores agrícolas, debido a las malas condiciones en que se realizaba la agricultura en la década de los ochenta y noventa en Chile, en un contexto de contracción y ajuste económico en dictadura, primero, y luego en la transición hacia la democracia:

«...en el caso de mi papá como agricultor, y para los agricultores en general, en ese tiempo todo estaba mal. Todo estaba mal. Lo que sembrabas costaba poca plata. Te pagaban poco y las producciones eran malas». (Gaete Padre). 
Del mismo modo, se describen las condiciones físicas que se requerían para trabajar en la tierra. «Todos los días con los caballos arando la tierra. Lo tenía chato el día a uno. [Uno] se bañaba y a acostarse». (Pradenas Padre).

La construcción de la subjetividad implica una relación con el entorno, ya sea biológico, meteorológico, sociológico o cultural (Morin, 1998: 70). La modernización cumplió un rol importante en la experiencia y percepción de los agricultores sobre el trabajo en el campo, sobre todo en los padres de las familias que participan en este estudio. De esta forma, las mejoras técnicas de la época que fueron incorporándose paulatinamente a la producción agrícola lograron, en parte, reducir el esfuerzo físico necesario para trabajar. Sin embargo, el esfuerzo asociado a la labor campesina se sigue manifestando en los discursos de estas familias, lo que influye de alguna manera en que sus hijos e hijas miren con especial interés otros tipos de labores asociadas a la ciudad. En este sentido, se observa una valoración positiva sobre el impacto de estas técnicas en la producción y sus efectos en el trabajo: «En nuestros tiempos era muy sacrificada la agricultura, era un trabajo bruto pero abora no, cualquier agricultor tiene un tractor y ara la tierra». (Pradenas Padre).

Sin embargo, los datos muestran que, en relación a la dimensión simbólica del trabajo, tanto para los trabajadores agrícolas como para los dueños de predio, si bien comparten ciertas valoraciones sobre los perfeccionamientos técnicos en la agricultura, se diferencian en el sacrificio que se requería para trabajar, además de las condiciones familiares y económicas en las que se insertaron. En este sentido, las entrevistas se evidencian las condiciones de extrema pobreza material de los temporeros ${ }^{3}$ en Chile, así como la incertidumbre y los abusos laborales sufridos durante la «modernización» vivenciada en dictadura y en la década posterior (Bengoa, 1996).

Por otra parte, un elemento llamativo resulta de la conversación con Ángel Céspedes, para quien «la agricultura sigue siendo la misma» (Céspedes Padre). Estas representaciones en apariencia contradictorias sobre el trabajo agrícola, creemos que se deben posiblemente a la naturaleza progresiva del proceso de cambio, que se cristaliza en la dicotomía entre la continuidad y la permanencia de un ethos campesino, y por otro lado, a las profundas transformaciones técnicas y sociales en las que se desarrolla la agricultura. Este ethos se instala en una narrativa dialógica entre individuo y sociedad, donde es la cultura la que se «recrea a sí misma en cada sujeto y cada sujeto da cuenta de ella» (Vargas, 2003: 74) a través de la comunicación, de un relato de sí mismos.

\subsection{Cambios intergeneracionales entre padres e hijos/as en torno a la agricultura}

Este apartado busca dar cuenta de aquellos factores que inciden en los cambios a nivel micro-sociológico, es decir, aquellos elementos que producen cambios a nivel individual en los jóvenes y sus aspiraciones. En este contexto, se hace relevante contrastar las percepciones acerca del trabajo en el campo, a partir de la visión que tienen los hijos, como también los cambios que han podido observar sus padres en las condiciones técnicas del trabajo. Destacamos aquí la relevancia de las significaciones que se construyen a partir del relato de sí mismo y del entorno social de los sujetos entrevistados.

De esta manera, y considerando que la «subjetividad se estructura a partir del lugar que ocupa el sujeto en la sociedad, y se organiza en torno a formas específicas de percibir, de sentir, de pensar y de actuar sobre la realidad» (Gil, 2007: 642), se observa en las entrevistas que esta posición es distinta

Trabajadores agrícolas de temporadas. 
de acuerdo al contexto que vivenciaron tanto padres como hijos/as. Para algunos de los hijos/as, la dimensión simbólica sobre lo rural está estrechamente relacionada a lo técnico, asimilando una pérdida de cultura y tradiciones campesinas debido a los avances tecnológicos. Sin embargo, al mismo tiempo que se lamenta esa pérdida, se reconocen los beneficios físicos que conlleva la incorporación de nuevas tecnologías en la agricultura.

«Entonces para mí, románticamente digo, se están perdiendo muchas cosas y yo sé que incluso, mi papá no me lo ha dicho así, pero yo sé que a él le duele poner riego tecnificado porque es fome $e^{4}$ poner una cuestión y que se riegue sola. Igual tiene su magia ponerse las botas, y no siento que se sienta cansado con ese trabajo». (Céspedes Hija).

Si bien el trabajo asalariado agrícola no es, en estricto rigor, una actividad laboral formalmente familiar, sí encontramos que, en todos los casos, los hijos/as han trabajado ocasionalmente junto a sus padres. Estas labores ocasionales son expresadas discursivamente como una ayuda al padre y una forma de alivianar la carga de su trabajo:

«Si, he trabajado con mis papás aqui, pero, por ejemplo, en las cortas de tomates durante las vacaciones o cosas así, pero es para cubrir mis gastos nomás y para ayudarles a mis papás creo yo. Pero trabajo asi afuera, no, nunca». (Gómez hija).

Las nociones subyacentes al trabajo, y cómo entran en cuestión al momento de expresar las experiencias de los hijos en la agricultura, están culturalmente situada en un contexto específico, y como dijimos, en el sistema capitalista se presenta en su forma asalariada. De esta manera, si se es minucioso, se puede señalar que para los hijos/as de los agricultores el sentido del trabajo como tal, se aproxima más a la concepción socialmente hegemónica del trabajo individual (salario, horario, jefes), y se aleja de la concepción ideal campesina en torno a la composición familiar del trabajo agrícola.

«... solamente he trabajado con mi papá, pero no sé si en el sentido de un trabajo. Ir a una entrevista, etc. Porque la experiencia laboral con mi papá es por una semana, o un mes. Abora últimamente no, desde que estoy en la universidad ya no». (Olmedo hijo).

Aquí se identifica el problema central de las familias agricultoras, que es el abandono de la actividad agrícola por la ausencia de traspaso generacional, y el ingreso de los y las jóvenes a la educación superior como medio para la realización de sus proyectos de vida. Además, aparece la influencia de la familia, en este caso la experiencia de los padres, como un elemento relevante en la representación que las y los hijos tienen de lo que es el trabajo agrícola, es decir, que los aspectos subjetivos de los hijos con respecto al trabajo agrícola están relacionados con experiencias que no fueron vividas directamente por ellos, sino que les han sido transmitidas por sus padres, dando forma a la significación del trabajo agrícola.

Esta idea de sacrificio es asumido a través de la socialización primaria, reproducida principalmente por breves periodos en que los jóvenes desempeñan labores en el campo, aunque sean vistas como una «ayuda» mínima por parte de ellos a sus padres. Será en esta socialización que «el individuo sujeto a esos discursos es sujeto de prácticas de sumisión a los mismos, pues la socialización es el proceso pasivo por el cual el individuo internaliza el orden socio-estructural y lo hace subjetivamente suyo, se

4 Aburrido, algo fuera de lugar. 
somete, de alguna forma» (Venegas, 2017: 26). Sin embargo, los campos de posibilidad de este sujeto lo encauzan hacía otros proyectos que surgen para sí, principalmente en virtud de distintas estructuras que entran en interacción con el individuo, destacando el papel de la universidad en ello, como se analiza a continuación.

\subsection{Aspiraciones juveniles: la universidad y «mi mejor vida»}

Las aspiraciones responden a los intereses, valores y convicciones que van entretejiéndose a través de la subjetividad, es decir, a través de aquellas experiencias que nutren y dan significados a los relatos personales. Visto de esta forma, diríamos que «la subjetividad no es algo preexistente, sino que se hace en el acto discursivo, en una narración de sí mismo, del otro y del mundo, de su pasado, presente y futuro en el aquí y ahora de la palabra, en las narrativas de los otros». (Vargas, 2003: 6).

En este sentido, si bien existe una valoración y reconocimiento por parte de los hijos/as en cuanto al trabajo de sus padres en la agricultura, éstos orientan sus proyectos de vida hacia otros trabajos cualificados a los que se accede a través de la formación en la educación superior, suponiendo en el caso contrario una situación desfavorable.

Es menester señalar que tanto las familias propietarias como las no propietarias manifiestan similares alusiones a las aspiraciones y a la importancia de la educación formal, además de constatar una situación análoga entre hombres y mujeres, padres e hijos. Para los padres, la educación superior tiene un contenido simbólico importante al relacionarlo con su propia experiencia de vida. Esta condición surge como una posibilidad de acceder a mejores condiciones de la que tuvieron ellos, que en su mayoría no fueron apoyadas por sus padres.

Para los padres, la educación superior de los hijos es un asunto importante al relacionarlo con su propia experiencia de vida. Esta condición surge como una posibilidad de acceder a mejores condiciones de la que tuvieron ellos, que en su mayoría no fueron apoyadas por sus padres: «Todos los que hemos trabajado en lo que es la tierra, no queremos que nuestros hijos pasen lo mismo». (Tapia padre)

Existe, por lo tanto, una mirada negativa por parte de los padres hacia una posible continuidad de su trabajo en el campo por parte de sus hijos. Esto coincide plenamente por lo señalado en Vivanco y Flores (2005), quien ubica entre las causas de esta problemática a la falta de recambio generacional, la transformación de los patrones culturales de las familias campesinas, cuyas visiones sobre la agricultura no la posicionan como un ejemplo a seguir, debido a que no genera bienestar económico ni social. Por lo tanto, los padres no quieren que sus hijos «vivan lo mismo que ellos». Esto también ha sido abordado en otras investigaciones, en cuanto a la relación con la paternidad de temporeros de la zona central de Chile, quienes ante la precariedad y pobreza material vivida por sus padres en el campo, buscan destinos distintos incentivando a sus hijos en la educación con un acento sacrificial sobre las acciones llevadas para tal propósito, y en ocasiones muestran exacerbación hacia un discurso paternal y al sobreconsumo (Caro, 2011).

En este sentido, en nuestra investigación hemos podido observar y destacar las formas en que es relatada la experiencia de los hijos en el proceso de educación formal desde el colegio hasta la universidad. El relato es enfático en las capacidades de sus hijos, así como en el compromiso de estos con su educación. Otro elemento es la comparación entre hijos de acuerdo a los roles de género. Se observa la inclinación de los padres hacia el reconocimiento de las capacidades y esfuerzos de las hijas en el 
proceso de educación, en comparación a sus hijos hombres: «... mi bija es súper buena en ese sentido. Desde primero de preparatoria que ella ha sido, por supuesto, no se ha echado ningún ramo y no ha perdido ninguna beca». (Céspedes padre).

Efectivamente, la socialización de género juega un papel central en la formulación de aspiraciones juveniles y la identificación con el trabajo académico (Gutman y Akerman, 2008). También la reproducción de estereotipos de género y de un modelo de maternidad dominante cumplen un papel importante en esto (Schoon, 2006), como se analiza más adelante.

La relevancia de las familias en cuanto a la educación universitaria de sus hijos e hijas es clave. Es importante añadir aquí la existencia de influencias que son abordadas de dos formas. Por un lado se reconoce una dependencia o cercanías de visiones en torno a la educación superior como una opción de vida, tanto para los jóvenes como para sus padres. Mientras que por otra parte, algunos hijos mencionan un cierto grado de independencia y responsabilidad personal en su decisión sobre sus estudios, sin que la influencia de los padres o la motivación de estos sean de gran relevancia: «Bueno, entré a estudiar sencillamente porque me gusta la independencia, valerme por mi misma, y sé que lo unico que me puede dar esa independencia son los estudio». (Sánchez hija).

Sin embargo, en general, las entrevistas muestran una heterogeneidad en las condiciones y trayectorias de vida que llevan a la generación joven a escoger la universidad. Las aspiraciones de los jóvenes están orientadas hacia la conformación de un proyecto de vida en primera instancia individual, que contempla en el corto plazo el goce de ciertas experiencias sobre la idea de conformar una familia. A partir de allí, se evidencian proyecciones laborales bastante optimistas con respecto a las posibilidades que ofrece una profesión. Otro elemento relevante es la importancia del reconocimiento social que implica trabajar como profesional:

«para mí, el día de mañana, yo quiero ser una mujer exitosa, ese es mi meta. Quizás no ser presidenta de la república, claro no ser grandes cosas; pero tal vez, trabajar en una empresa, donde yo sí sea reconocida. Donde diga que sí contribuye al desarrollo del país». (Gaete hija).

Es en este aspecto donde las entrevistas muestran la cuestión central con respecto a la individualización de las aspiraciones. Como se ve, el éxito y el reconocimiento están condicionados por las situaciones de ascenso laboral individual y no un proyecto colectivo en concreto. Sin embargo, también se señala que el reconocimiento esperado proviene de la contribución a otro sujeto colectivo, en este caso, un reconocimiento al «desarrollo del país». Por lo tanto, vemos que cualquier afirmación con respecto a la individuación resulta compleja integrando aspectos individuales y colectivos.

Ante esta situación, Los padres entienden que las opciones de sus hijos están sujetas a las características del mercado laboral en Chile, y a las circunstancias difíciles en que se encuentran para la proyección de nuevas generaciones en la agricultura. Los agricultores observan que la renovación generacional no se está produciendo, mientras los jóvenes encuentran otras opciones laborales fuera de la agricultura. Por otra parte, los jóvenes que, finalmente optan por el trabajo agrícola, encuentran numerosas dificultades para desarrollarse en este campo, debido a una escasa oferta de empleos, y bajos salarios. «... ahora no, la mayoría de los cabros les gusta trabajar en los supermercados. Aqui en Limache están llegando los supermercados: trabajos más limpios, más livianos y con mejores temperaturas». (Gómez padre). 
Con todo, salta a la luz un elemento que ha estado bastante presente en el estudio del campesinado, que es el factor relacionado al territorio. En este caso, hablamos de las nociones de lo rural o el campo. En efecto, las transformaciones del espacio rural en Chile están estrechamente relacionadas a los cambios en el trabajo agrícola. Por ejemplo, vemos como la llegada de supermercados y empresas de servicio traen consigo ofertas laborales que son bien vistas por los jóvenes, quienes encuentran mayor estabilidad y menos requerimiento físico a la hora de conseguir un salario.

En este contexto, las percepciones de los agricultores tienden a asumir estos cambios como perjudiciales para la agricultura en tanto trabajo y actividad. No solo es la urbanización entendida como la llegada de servicios y comercio, sino que también se menciona la llegada de condominios y parcelas de agrado ${ }^{5}$, que aprovechan los precios de suelo inferiores en sectores rurales para utilizarlos como territorios destinados a la vivienda: «Las parcelas de agrado han sido una destrucción para la agricultura». (Gómez padre).

En los hijos, existe una valoración positiva de la vida del campo, y se le atribuyen aspectos de tranquilidad y bienestar. Esta percepción del territorio rural se ve reflejado en sus proyectos de vida, al momento de pensar en qué lugar les gustaría vivir. Muestran una disposición positiva a vivir en sectores rurales, y negativa ante la posibilidad de vivir en grandes ciudades:

«Nosotros ya tenemos todo definido: pensamos que nos vamos a casar, pero cerca. Qué tan cerca, no sé si acá en San Pedro, pero no al medio de una ciudad. No en Viña, no en Valparaíso, no en Santiago. Yo creo que también con lo que piensa él, tener una casa con árboles, con plantas, con no sé qué, entonces los dos pensamos en cosas cercanas como Olmué, algo tranquilo. Incluso acá en San Pedro, pero no encima de nuestras familias». (Céspedes hija).

Podemos ver los matices que surgen en este vínculo con el entorno familiar y el proyecto de vida de los jóvenes. El hecho de que manifiesten una disposición a disfrutar personalmente de los beneficios económicos de los estudios superiores, no implica en absoluto un rechazo a la idea de un proyecto familiar, más bien se trata de relegar estos proyectos para cuando existan las condiciones óptimas para ello.

\subsection{Postergación de la paternidad y maternidad de la nueva juventud}

Las entrevistas muestran también la visión de que la maternidad y paternidad implican una responsabilidad que da término a la juventud. En este sentido, la paternidad y la maternidad vistas como marcadores vitales, se convierten en hitos que marcan el paso de la juventud a la adultez. Esta delimitación simbólica sobre el paso de una etapa de la vida a otra, tiene una inspiración objetiva que reconoce los gastos y responsabilidades implicadas en la paternidad y la maternidad, además de las dificultades que presentan estas condiciones para las exigencias del mercado laboral actual.

Esta visión es similar en hombres y mujeres, aunque se puede pensar en que el género sí tiene un rol importante en la asimilación de esta percepción, principalmente si se tiene en cuenta la adscripción de ciertos roles por parte de los hijos dentro de la estructura familiar:

"Algún día voy a tener familia, pero no, no todavía. No voy a estar cinco años estudiando para tener que empezar a trabajar para otra persona. Asi como para tener que alimentar a mi bijo, y yo zqué voy a ver?, ;no!, lo siento». (Sánchez hija).

5 Los condominios son conjunto de viviendas agrupadas en un sector específico, generalmente cuentan con servicios de vigilancia, entre otros. Las parcelas de agrado son terrenos ubicados en sectores de suelo agrícola, subdivididos para ser utilizado para viviendas. Muchas parcelas de agrado están agrupadas como condominios. 
La maternidad es un asunto importante para las entrevistadas al momento de abordar sus proyectos de vida. Para ellas, la maternidad representa una circunstancia problemática para la realización de sus objetivos personales, principalmente en lo referido a las exigencias económicas que trae la crianza de hijos. Como se señala anteriormente, el goce individual de los frutos del esfuerzo y el tiempo invertido durante los estudios está por encima de la formación de un grupo familiar. No obstante, esta circunstancia es vista como una etapa particular de la vida para, posteriormente, dar paso al proyecto familiar, es decir, se trata principalmente de una postergación.

Este fenómeno ha sido ampliamente investigado a distintos niveles de análisis. El más general, señala que la modernización de la sociedad, el fin de la familia tradicional y retroceso de la autoridad paternal dentro de la familia, trajo una mayor participación económica de la mujer. Esto a su vez, está relacionado con una integración a la educación que amplía la autonomía y autovaloración de la mujer en la sociedad (Jelin, 1998).

En efecto, existe una relación entre las condiciones socioeconómicas de la mujer y la postergación de la maternidad, como ha sido constatado en algunos países de Latinoamérica como México y Chile (Montilva, 2008); no obstante, esto se ha centrado en los sectores urbanos (metrópolis) de dichos países. En los sectores rurales, comúnmente se han estudiado las transformaciones de la familia tradicional campesina con el ingreso de las mujeres al mercado laboral, que han tenido efectos similares. A saber, transformaciones simbólicas en el seno de la familia que, a diferencia de las mujeres de generaciones anteriores, no se perfilan bajo identidad definida exclusivamente por maternidad.

Sin embargo, en estudios realizados con mujeres del segmento entre 30 y 40 años en Chile en zonas rurales, se observa que la maternidad mantiene la preocupación por la educación de los hijos, replicando el lema de los padres de «para que no sean como uno» (Valdés y Villagrán, 2015). Por otra parte, hay quienes señalan que para las mujeres rurales, el trabajo trae consigo un empoderamiento y agencia femenina que, sin embargo, no tiene las funciones de reemplazar a las familias sino de complementarlas (Villagrán et al., 2014). Si contrastamos esta última afirmación, podemos señalar una visión de una relativa incompatibilidad entre trabajo y familia, no solo en lo referido a los aspectos económicos, también sobre un 'instinto maternal' que, en su caso, 'no existe'. Cabe señalar, que la edad cumple un rol fundamental en la conformación de representaciones de maternidad que, lejos de acercarnos a un determinismo etario, nos obliga a realizar comparaciones teniendo en cuenta este elemento. Sin duda, este es un tema de amplio proceder que merece una revisión más amplia que, sin embargo, queda fuera de los márgenes de esta investigación, cuyos datos muestran la aparición de estas perspectivas sobre la maternidad y la relación que tienen con la educación y las aspiraciones de jóvenes en contextos rurales.

\section{Conclusiones}

Este artículo tenía como objetivo analizar la dimensión subjetiva que subyace al abandono de la agricultura en hijos e hijas de familias agrícolas, así como la configuración de las aspiraciones intergeneracionales a partir del acceso a la educación superior. Tras analizar los datos procedentes de las entrevistas de esta investigación, cabe concluir con algunas ideas principales.

En primera instancia, es posible señalar que los datos muestran que ambas dimensiones de ese análisis se encuentran permeadas por una dinámica social de corte neoliberal que ha penetrado lo subjetivo, influyendo en las aspiraciones familiares en contextos donde el trabajo agrícola sigue presente en la generación de progenitores en el ámbito rural chileno. 
Las aspiraciones juveniles investigadas en este estudio están relacionadas, en términos sociales, con la masificación de la educación superior y el deterioro de las condiciones de trabajo agrícola en las zonas rurales en Chile. Pese a existir diferencias entre los casos vistos, como la propiedad de un predio y experiencias pretéritas en la agricultura, hay transversalidad en la formación de estas aspiraciones. En términos subjetivos, la individualización de la vida y el trabajo también se relacionan con propuestas teóricas que describen un escenario de sociedad del riesgo en el contexto del desarrollo del neoliberalismo. Como se ha podido observar, la influencia y experiencias de los padres tienen un impacto importante en las aspiraciones de las y los jóvenes, ya sea en términos de dependencia familiar o autonomía individual, del mismo modo que no se observa una desruralización completa, al preferir el medio rural sobre la ciudad para sus proyectos de vida.

Por otra parte, es importante entender los procesos subjetivos subyacentes al abandono de la agricultura puesto que, si bien existen datos que comprueban esta tendencia, se encuentran ante la dificultad de generar una descripción general del fenómeno debido a la alta heterogeneidad de este segmento de la población en Chile. Si bien, en las entrevistas realizadas se tratan temas relevantes para el debate en este país en cuanto a la aproximación de la realidad de la juventud rural, los datos no ofrecen información sobre otros puntos de igual relevancia como los roles de género, atendiendo a las especificidades del territorio y la historia. El estudio sobre esos fenómenos, además, podría ser útil en cuanto a la formulación de políticas públicas destinadas a la agricultura, persistiendo hasta el día de hoy desafíos pendientes en cuanto a la tipificación de la población agrícola con el fin de formular estrategias pertinentes y beneficiosas para la población (INDAP, 2014).

Como bien se ha investigado tanto en Estados Unidos, como Europa y Latinoamérica, las aspiraciones juveniles tienen una estrecha relación con las expectativas creadas por el ingreso en la universidad. Conseguir un título universitario no solo es visto como una condición más favorable para conseguir mejores empleos y salarios, también se consideran aspectos relacionados al reconocimiento social de «tener una carrera».

Estas aspiraciones no solo son compartidas por sus padres, sino que también son fortalecidas, recreando de esta forma expectativas de vida sobre sus hijos con los recuerdos de su propia juventud, y la necesidad que tuvieron de trabajar en la agricultura en condiciones difíciles. Por esta razón, las aspiraciones en torno a la educación superior no son únicamente juveniles, lo son también en un sentido simbólico y afectivo, familiares.

El trabajo agrícola para los sujetos entrevistados es reconocido por las desventajas que representa con respecto a los trabajos no agrícolas, debido al gran esfuerzo físico que requiere para llevarse a cabo. De igual forma, persiste una visión romántica y afectiva hacia el trabajo y el predio familiar que fue adquirido por generaciones anteriores. Sin embargo, estas ambivalencias ocurren únicamente en las familias propietarias, mientras que en el caso de asalariados agrícolas la afectividad hacia el trabajo disminuye al recordar las experiencias de tiempos pasados: abusos laborales, extrema pobreza y condiciones difíciles de trabajo dotan de una carga emocional las aspiraciones educacionales, bajo el repetido lema de «no quiero que mis hijos pasen por lo mismo».

A pesar de que existe una comunión entre estos aspectos, las aspiraciones de los hijos son proyectadas en opciones de vida relativamente individualizadas, en el sentido de que la posibilidad de formar familias o tener hijos son vistas como una dificultad para el goce de la independencia y mejor condición socioe- 
conómica. Esto también tiene una similitud con la visión de los padres; para ellos, el tener hijos fue un marcador vital que «termina» la juventud y requiere de responsabilidades. No obstante, para los hijos no existe un rechazo completo a la idea de formar familias sino que, más bien, ésta es aplazada. Otro elemento destacable es la valoración positiva referida a la vida en zonas rurales, atribuyendo a este aspecto tranquilidad y bienestar.

En este sentido, las aspiraciones y expectativas de vida mantienen ciertas características descritas como una subjetividad neoliberal con una presencia alta de elementos individualistas. Si bien, existen prácticas de resistencia en el interior de las familias, lo que impide una valoración positiva del trabajo agrícola en el campo por parte de los hijos e hijas de estas familias, se observa también una alta valoración individual que apunta al crecimiento y éxito económico propio de sociedad neoliberales, desde donde podemos establecer un continuum que reproduce este modelo dominante. $\mathrm{Al}$ mismo tiempo, existe una resistencia en tanto persistencia de un arraigo al campo y la vida rural, así como un reconocimiento, aunque postergado, del proyecto familiar. De esta manera, la idea que tienen los jóvenes del campo es vista como una extensión de elementos de cercanía con sus padres, desde donde surge un respeto por su labor, pero en ningún caso forma parte del proyecto de vida y sustento económico para estos jóvenes. Podemos en consecuencia, establecer que existen algunos factores que inciden en este fenómeno; por un lado está la familia, que con su discurso de sacrificio limita el interés de sus hijos por el trabajo agrícola; por otro lado encontramos el proceso de urbanización de estos territorios, lo que sumado a una creciente consumo cultural asociado a las tecnologías, facilitan la absorción de valores culturales propios de las ciudades, como los son la educación, bienes y servicios, finalmente; la educación aparece como un motor de desarrollo personal, a partir del cual es posible integrarse plenamente los valores sociales de la ciudad, alejándose de la cultura rural. En definitiva, estamos ante un proceso de transición de los jóvenes en el campo chileno, quienes proyectan sus vidas con metas claras hacia el camino que buscan trazar.

\section{Referencias bibliográficas}

Archetti, Eduardo (1977): "El proceso de capitalización de campesinos argentinos". La terre et les paysans en Amérique Latine, 28, 123-140.

Ball, Stephen J.; Youdell, Deborah (2008). La privatización encubierta de la educación pública. Instituto de Educación, Universidad de Londres.

Ball, Stephen J. (2013): “Care of the Self, Resistance and Subjectivity under Neoliberal Governmentalities". Critical Studies in Education, 54.

Ball, Stephen J. (2015): “Subjectivity as a Site of Struggle: Refusing Neoliberalism?”. British Journal of Sociology of Education, 37 (8), 1129-1146 https:// doi.org/10.1080/01425692.2015.1044072

Bengoa, José (1996): “Campesinado y Mercosur”. Ediciones sur, 12.

Beck, Ulrich (1998). La sociedad del riesgo: Hacia una nueva modernidad. Barcelona, España: Editorial Paidós.

Bertrán, Gloria; Corbalán, Javier (1990). Juventud Rural, capacitación y empleo: Diagnóstico socio-laboral. Santiago de Chile: Editorial ARGÉ.

Bourdieu, Pierre (2010). "Comprender”, en La miseria del mundo. Fondo de Cultura Económica: Buenos Aires. 
Cairns, Kate (2013): “The subject of Neoliberal Affects”. The Canadian Geographer, 57 (3), 337-344.

Canales, Manuel (2006). Metodologías de la investigación social: Introducción a los oficios. Santiago de Chile: LOM.

Caro, Pamela (2011): “Los padres ‘de abajo': La paternidad en los temporeros agrícolas”. Centro de Estudios para el Desarrollo de la Mujer, 2,123-142.

CEPAL (1994). Juventud rural: Modernidad y democracia en América Latina. Santiago de Chile: CEPAL.

Colodro, Max (1995). Juventud Rural: percepciones sobre el mundo laboral (tesis doctoral). Santiago de Chile: Universidad de Chile.

Díaz, Cecilia y Durán, Esteban (1986). Los jóvenes del campo: una identidad fragmentada. Santiago de Chile: Grupo de Investigaciones Agrarias.

Díez-Gutiérrez, Enrique Javier (2015): “La educación de la nueva subjetividad neoliberal”. Revista Iberoamericana de Educación, 68 (2), 157-172.

De la Maza, Gonzalo (1993). Los jóvenes temporeros de la V región: un abordaje cualitativo. Santiago de Chile: Estudios Del INJUV.

Gonzalez, Yanko (2003). “Juventud Rural: trayectorias teóricas y dilemas identitarios". Revista Nueva Antropología, 19 (63), 153-175.

Guber, Rosana (1994). La etnografia: método, campo y reflexividad. Bogotá: Editorial Norma.

Morrison Gutman, Leslie and Akerman, Rodie (2008). Determinants of Aspirations. Londres: Centre of Research on the Wider Benefits of Learning.

Harvey, David (2007). Breve historia del neoliberalismo. Recuperado de https://teoriaeconomicatercersemestreri.files.wordpress.com/2012/09/breve-historia-del-neoliberalismo-de-david-harvey1.pdf

Hernández, Ronerto (1994): “Teorías sobre el campesinado: Una evaluación crítica”. Revista Chilena de Antropología, 12, 179-200.

INDAP. (2014). Lineamientos estratégicos 2014-2018: por un Chile rural inclusivo. Chile: INDAP.

Jelin, Elizabeth (1998). Pan y afectos: la transformación de las familias. Buenos Aires: Fondo de Cultura Económica.

Kay, Cristóbal (1997): “La cuestión agraria y el campesinado en Chile hoy”. Debate Agrario, 27, 79-110.

Kenway, Jane (1990): “La educación y el discurso político de la Nueva Derecha” en Stephen J. Ball (Ed.): Foucault y la educación: Disciplinas del saber. Madrid: Ediciones Morata.

Montilva, Maira (2008): "Postergación de la maternidad de mujeres profesionales jóvenes en dos metrópolis latinoamericanas". Utopia y praxis Latinoamericana, 13 (41), 69-79.

Morin, Edgar (1998): “La noción del sujeto" en Dora Fried Schnitman (ed.): Nuevos Paradigmas, Cultura y Subjetividad. Buenos Aires: Editorial Paidós.

Quaglia, Russel and Cobb, Casey (1996): “Toward a Theory of Student Aspirations". Journal of Research in Rural Education, 12 (3), 127-132.

Rodríguez, Emilio (2012): “La educación superior en Chile y el rol del mercado: ¿Culpable o inocente?” Revista chilena de ingeniería, 20 (1), 126-135. 
Sautu, Ruth (2003). Todo es teoría. Objetivos y métodos de investigación. Buenos Aires Argentina: Editorial Lumiere.

Sherwood, Richard A. (1989): “A Conceptual Framework for the Study of Aspirations”. Journal Research in Rural Education, 6 (2), 61-66.

Schoon, Ingrid (2006). Risk and Resilience: Adaptations in Changing Times. Cambridge: Cambridge University Press.

Schwiter, Karin (2012): "Neoliberal Subjectivity-Difference, Free Choice and Individualized Responsibility in the Life Plans of Young Adults in Switzerland”. Geographical Helvetica, 68, 153-159.

Valdés, Alberto y Foster, William (2015). La reforma Agraria en Chile: Historia, efectos y lecciones. Santiago de Chile: Ediciones UC.

Valdés, Ximena y Rebolledo, Loreto (2015): “Géneros, generaciones y lugares: cambios en el medio rural de Chile Central". Polis Revista Latinoamericana, 14 (42), 491-513.

Vargas, Lilia Esther (2003). ¿La subjetividad del sujeto o el sujeto de la subjetividad? Tras las huellas de la subjetividad. México DF: UAM.

Venegas, Mar (2017): “Devenir sujeto. Una aproximación sociológica”. Convergencia revista de ciencias sociales, 73, 13-36.

Soto Villagrán, Paula; Fawaz Yissi, Julia y Vallejos, Rosana (2014): “Género, Trabajo y Familia. Discursos y prácticas de empoderamiento de mujeres rurales". Estructuras, dinámicas y significados del trabajo femenino: Nuevas perspectivas espaciales, estructurales y simbólicas. Ponencia llevada a cabo en la Universidad del Biobío.

Vivanco, Manuel y Flores, Rodrigo (2005): "Entre la naturaleza y el mercado: El caso de una organización familiar campesina. Santiago de Chile". Revista de Sociología, 19, 171-182.

\section{Notas biográficas}

Hernán Allendes Sandoval es Doctor en Ciencias de la Educación por la Universidad de Granada. Profesor Agregado de la Facultad de Agronomía de la Pontificia Universidad Católica de Valparaíso. Cátedras producción de Hortalizas durante 26 años, (1991 a la fecha). Línea de investigación: nuevos escenarios rurales. Consultor para pequeños y medianos productores de hortalizas a través de programas del Ministerio de Agricultura de Chile.

Enrique Rivera García es Profesor Titular de Universidad del Departamento de Didáctica de la Expresión Musical, Plástica y Corporal de la Facultad de Ciencias de la Educación de la Universidad de Granada. Línea de investigación: Educación, Corporalidad y Transformación Social. Especialista en Metodología de Investigación Cualitativa asistida por software de análisis y producción de información. 